\title{
Pairing of Lyapunov Exponents for a Hard-Sphere Gas under Shear in the Thermodynamic Limit
}

\author{
Debabrata Panja* and Ramses van Zon ${ }^{\dagger}$ \\ * Instituut Lorentz, Universiteit Leiden, \\ Postbus 9506, 2300 RA Leiden, The Netherlands. \\ $\dagger$ Chemical Physics Theory Group, Chemistry Department, University of Toronto, \\ 80 St. George St., Toronto, Ontario M5S 3H6, Canada.
}

\begin{abstract}
We consider a dilute gas of hard spheres under shear. We use one of the predominant models to study this system, namely the SLLOD equations of motion, with an iso-kinetic Gaussian thermostat in between collisions, to get a stationary total peculiar kinetic energy. Based on the previously obtained result that in the non-equilibrium steady state and in case the number of particles $N$ becomes large, the coefficient of dynamical friction representing the iso-kinetic Gaussian thermostat for the SLLOD dynamics fluctuates with $1 / \sqrt{N}$ fluctuations around a fixed value, we show on analytical grounds that for a hard sphere gas at small shear rate and with a large number of spheres, the conjugate pairing of the Lyapunov exponents is expected to be violated at the fourth power of the constant shear rate in the bulk.
\end{abstract}

PACS Numbers: 05.20.-y, 05.45.-a, 05.60.Cd

\section{INTRODUCTION}

Non-equilibrium molecular dynamics (NEMD) simulations of Navier-Stokes equations have been used to study the shear viscosity properties of fluids for a long time. To study the coefficient of shear viscosity in Navier-Stokes equations, a carefully chosen periodic boundary condition in NEMD simulations is enough to drive the system out of equilibrium. Based on these ideas, in the beginning days of the development of this subject, an algorithm was constructed from simple Newtonian equations of motion using a periodic boundary conditions, the so-called LeesEdwards boundary conditions [1]. However, it was soon realized that in the absence of an explicit dependence on the shear field in this algorithm, one could not make an appropriate connection with the Green-Kubo relations, and therefore, it was difficult to deal with from a theoretical point of view. As a remedy, some other algorithm with an explicit dependence on the shear field was called for, and the DOLLS and the SLLOD algorithms were born.

The main idea behind the DOLLS and the SLLOD algorithm is an explicit dependence on the shear field, $\gamma$. The DOLLS algorithm was implemented first [2]. The SLLOD equations of motion were proposed soon after [3], and are now preferred because they are equivalent to the boundary driven method [4]. Both algorithms have to be supplemented by a thermostat, which continuously removes the energy generated due to the work done on the system by the shear field such that an nonequilibrium steady state (NESS), homogeneous in space, can be reached.

In this paper, we will look at the SLLOD equations of motion for a gas of hard spheres from the point of view of dynamical systems. The Lyapunov exponents of a system of particles, obeying the SLLOD equations of motion and mutually interacting by means of WCA potential with an iso-kinetic Gaussian thermostat, was first studied by Morriss [5]. The study showed that the shear viscosity can be obtained from the sum of all the Lyapunov exponents. The simulation results in Ref. [5] also indicated that once the Lyapunov exponents are arranged in ascending order of magnitude, the sum of the largest and the smallest, the sum of the second largest and the second smallest and so on, were the same. The phenomenon of such pairing of the Lyapunov exponents is known as the Conjugate Pairing Rule, or the CPR. Since it is in general difficult to calculate all the individual Lyapunov exponents of a system, an extensive theoretical study soon ensued to understand the CPR for the Lyapunov exponents of systems obeying the SLLOD equations of motion. Evans and co-workers investigated this point [6] to conclude that the Lyapunov exponents pair exactly for general inter-particle potentials and all $\gamma$. In a follow-up work, Sarman et al. [7 carried out simulation studies in support of Ref. [6].

In the next few years, the connection between the dynamical systems theory and statistical mechanics saw a surge of interest. Some situations were found, where it was possible to prove that the CPR is satisfied exactly 811. The status of the CPR for the SLLOD and the DOLLS dynamics was revisited. For a system of particles obeying the SLLOD and the DOLLS dynamics with a WCA inter-particle potential and arbitrary $\gamma, \mathrm{CPR}$ was reported to be violated on the basis of simulation results [12,13, but recently it was shown that this claim is based on an erroneous analysis of the Lyapunov spectrum [14]. However, for these two systems, no attempt of a theoretical understanding about the nature of an approximate 
CPR has been carried out so far. In this paper, we address and attempt to clarify these issues. We find that for a dilute gas of hard spheres obeying the SLLOD dynamics, where the masses and the radii of the spheres are not necessarily the same, and the total peculiar kinetic energy is kept constant by applying the iso-kinetic Gaussian thermostat in between collisions, the CPR is violated at the most at $O\left(\gamma^{4}\right)$, for small $\gamma$, in the thermodynamic limit. 15] Our analysis is based on the key idea that the coefficient of friction representing the isokinetic Gaussian thermostat for a dilute gas of particles mutually interacting by means of a short-ranged potential and obeying the SLLOD dynamics with a small shear rate, in the NESS, reaches a fixed value in the thermodynamic limit, with $1 / \sqrt{N}$ fluctuations, where $N$ is the number of particles. [16].

The structure of the paper is the following: in Sec. II, we describe equations of motion for the SLLOD dynamics, define the Lyapunov exponents, and discuss the sufficient conditions for an exact CPR. In Sec. III, we demonstrate how the coefficient of friction representing the isokinetic Gaussian thermostat is expected to reach a fixed value at the thermodynamic limit, with $1 / \sqrt{N}$ fluctuations. In Sec. IV, we present the explicit calculations and discuss the status of an approximate CPR. To make the calculations In Secs. II-IV simple, we assume that each of the gas particles has a unit mass. Finally, we end this paper with discussions on possible generalizations, including the generalization to the case when the masses of the gas particles are arbitrary, in Sec. V.

\section{THE SLLOD EQUATIONS OF MOTION FROM A VIEWPOINT OF DYNAMICAL SYSTEMS}

\section{A. Equations of Motion}

The SLLOD equations of motion describe the dynamics of a collection of $N$ particles constituting a fluid with a macroscopic velocity field $\mathbf{u}=\gamma y \hat{\mathbf{x}}$ (i.e., the gradient of the $x$-component of the macroscopic fluid velocity $\mathbf{u}$ in the $y$-direction is $\gamma$ ). For simplicity, each gas particle is assumed to have a unit mass. The specific form of the SLLOD equations of motion for the $i$-th particle, in terms of its position $\mathbf{r}_{i}$ and peculiar momentum $\mathbf{p}_{i}$, is given by

$$
\dot{\mathbf{r}_{i}}=\mathbf{p}_{i}+\gamma y_{i} \hat{\mathbf{x}}, \quad \dot{\mathbf{p}}_{i}=\mathbf{F}_{i}-\gamma p_{i y} \hat{\mathbf{x}}-\alpha \mathbf{p}_{i} .
$$

where $\mathbf{F}_{i}$ is the force on the $i$-th particle due to the other particles in the system. The peculiar velocity of a particle is defined as its velocity with respect to the velocity of the flow at its location and the peculiar momentum of a particle is the product of its mass and its peculiar velocity. The value of $\alpha$, the coefficient of friction representing the iso-kinetic Gaussian thermostat in Eq. (2.1), is chosen such that the total peculiar kinetic energy of the system, $\sum_{i} p_{i}^{2} / 2$, is a constant of motion, i.e.,

$$
\alpha=\frac{\sum_{i=1}^{N}\left(\mathbf{F}_{i} \cdot \mathbf{p}_{i}-\gamma p_{i x} p_{i y}\right)}{\sum_{i=1}^{N} p_{i}^{2}} .
$$

The SLLOD equations of motion, without the dissipative term $-\alpha \mathbf{p}_{i}$, cannot be derived from a Hamiltonian (unlike the DOLLS equations of motion).

We will use the equations of motion exclusively in terms of the particles' positions $\mathbf{r}_{i}$ and laboratory velocity $\mathbf{v}_{i}$. This introduces the change of variable from $\mathbf{p}_{i}$ to $\mathbf{v}_{i}=\mathbf{p}_{i}+\gamma y_{i} \hat{\mathbf{x}}$ in Eq. (2.1), which can then be written as

$$
\dot{\mathbf{r}}_{i}=\mathbf{v}_{i}, \quad \dot{\mathbf{v}}_{i}=\mathbf{F}_{i}+\alpha \gamma y_{i} \hat{\mathbf{x}}-\alpha \mathbf{v}_{i}
$$

In the present context, the gas particles are hard spheres of arbitrary radii. This reduces the dynamics of the gas particles to an alternating sequence of flight segments and instantaneous binary collisions. During a flight, the dynamics of the gas particles is therefore described by Eqs. 2.2 2.3) with $\mathbf{F}_{i}=0$. As far as the dynamics in collisions is concerned, it is possible to derive the limiting behavior of the iso-kinetic Gaussian thermostat as $\mathbf{F}_{i} \rightarrow \infty$ [17, but this leads to rather complicated collision rules. For the purpose of simplicity, in this paper, we choose to apply the iso-kinetic thermostat only in between collisions. Thus, at an instantaneous collision between the $i$-th and the $j$-th sphere $(i, j=1,2, . ., N ; i \neq j)$, the post-collisional positions and laboratory momenta ( + subscripts) are related to their pre-collisional values ( - subscripts) by

$$
\begin{aligned}
\mathbf{r}_{i+} & =\mathbf{r}_{i-}, \quad \mathbf{r}_{j+}=\mathbf{r}_{j-}, \\
\mathbf{v}_{i+} & =\mathbf{v}_{i-}-\left\{\left(\mathbf{v}_{i-}-\mathbf{v}_{j-}\right) \cdot \hat{\mathbf{n}}_{i j}\right\} \hat{\mathbf{n}}_{i j}
\end{aligned}
$$

and

$$
\mathbf{v}_{j+}=\mathbf{v}_{j-}+\left\{\left(\mathbf{v}_{i-}-\mathbf{v}_{j-}\right) \cdot \hat{\mathbf{n}}_{i j}\right\} \hat{\mathbf{n}}_{i j},
$$

while the positions and the velocities of the rest of the spheres remain unchanged. Here, $\hat{\mathbf{n}}_{i j}$ is the unit vector along the line joining the center of the $i$-th sphere to the $j$-th sphere at the instant of collision. Note that although in any particular collision, the peculiar kinetic energy changes over a collision, these changes are random, both in magnitude and sign, due to the randomness of the collision parameters, and hence it is quite likely that the system would reach a steady state, where the average change of peculiar kinetic energy would be zero.

To study the SLLOD dynamics as a dynamical system in three dimensions (the dimensionality does not affect our analysis), we form the $3 N$-dimensional vectors $\mathbf{R}=\left(\mathbf{r}_{1}, \mathbf{r}_{2}, \ldots, \mathbf{r}_{N}\right), \mathbf{V}=\left(\mathbf{v}_{1}, \mathbf{v}_{2}, \ldots, \mathbf{v}_{N}\right)$ and $\hat{\mathbf{N}}_{i j}$, whose $l$-th entry is given by $\hat{\mathbf{N}}_{i j}^{l}=\left(\delta_{l, i}-\delta_{l, j}\right) \hat{\mathbf{n}}_{i j} / \sqrt{2}(l=$ $1,2, \ldots, N)$. Using these new variables, we write the SLLOD equations of motion in the $\left(\mathbf{r}_{i}, \mathbf{v}_{i}\right)$ coordinates of $N$ hard spheres during a flight, Eq. (2.3), in a compact form 


$$
\dot{\mathbf{R}}=\mathbf{V}, \quad \dot{\mathbf{V}}=\alpha \gamma \mathbf{C R}-\alpha \mathbf{V} .
$$

Here, C is a $3 N \times 3 N$ matrix with $N \times N$ entries, each of which is a $3 \times 3$ matrix. In terms of the entry index $(l, m)$, in the $x y z$-basis, $\mathrm{C}_{l m}=\mathrm{c} \delta_{l m}(l, m=1,2, . ., N)$ and

$$
\mathrm{c}=\hat{\mathbf{x}} \hat{\mathbf{y}}=\left[\begin{array}{lll}
0 & 1 & 0 \\
0 & 0 & 0 \\
0 & 0 & 0
\end{array}\right] \text {. }
$$

At a collision between the $i$-th and the $j$-th sphere, the equations of motion are given by [11]

$$
\mathbf{R}_{+}=\mathbf{R}_{-}, \quad \mathbf{V}_{+}=\mathbf{V}_{-}-2\left(\mathbf{V}_{-} \cdot \hat{\mathbf{N}}_{i j}\right) \hat{\mathbf{N}}_{i j} .
$$

In our analysis hereafter, except for Sec. III, we will use only Eqs. (2.7-2.9) to describe the dynamics.

At this point, we introduce the following notations. A phase space point can be denoted as $\boldsymbol{\Gamma}=(\mathbf{R}, \mathbf{V})$. A linear transformation on phase space can be given as a $6 N \times 6 N$ matrix. Any such matrix $\mathrm{P}$ can be split in terms of four $3 N \times 3 N$ sub-blocks, for which we use the notation $\mathrm{P}^{[1]}, \mathrm{P}^{[2]}, \mathrm{P}^{[3]}$ and $\mathrm{P}^{[4]}$, such that

$$
P=\left[\begin{array}{ll}
P^{[1]} & P^{[2]} \\
P^{[3]} & P^{[4]}
\end{array}\right]
$$

Each sub-block $\mathrm{P}^{[i]}(i=1, \ldots, 4)$ itself can be divided in $3 \times 3$ sub-blocks again, where each sub-block can be identified by two indices $l$ and $m, l$ along the horizontal and $m$ along the vertical direction $(l, m=1,2, . ., N)$. Such a sub-block is denoted by $\mathrm{P}_{l m}^{[i]}$.

\section{B. Lyapunov Exponents for Hard-sphere Systems}

To calculate the Lyapunov exponents for hard-sphere systems, let us say that the system starts at time $t_{0}$ at a phase-space location $\boldsymbol{\Gamma}\left(t_{0}\right) \equiv\left(\mathbf{R}\left(t_{0}\right), \mathbf{V}\left(t_{0}\right)\right)$. Under time evolution, $\boldsymbol{\Gamma}(t)$ follows a trajectory in the $6 \mathrm{~N}$ dimensional phase space, which we call the "reference trajectory". The set of $N$ hard spheres would suffer a sequence of binary collisions on this trajectory. We also consider an infinitesimally displaced trajectory in the phase space, which starts at the same time $t_{0}$, but at $\boldsymbol{\Gamma}^{\prime}\left(t_{0}\right)=\boldsymbol{\Gamma}\left(t_{0}\right)+\delta \boldsymbol{\Gamma}\left(t_{0}\right)$. Under time evolution, $\boldsymbol{\Gamma}^{\prime}(t)$ follows another trajectory, always staying infinitesimally close to the reference trajectory. This trajectory we call the "adjacent trajectory". We also assume that the set of $N$ hard spheres on the reference and the adjacent trajectories suffer the same sequence of binary collisions. We denote the time evolution of the infinitesimal $6 N$ dimensional tangent vector $\delta \boldsymbol{\Gamma}(t)$ over time $\left(t-t_{0}\right)$ by the $6 N \times 6 N$ matrix $\mathrm{L}\left(t-t_{0}\right)$, i.e.,

$$
\delta \boldsymbol{\Gamma}(t)=\mathrm{L}\left(t-t_{0}\right) \delta \boldsymbol{\Gamma}\left(t_{0}\right) .
$$

The Lyapunov exponents are the possible exponential growth rates in time of $|\mathrm{L} \hat{\boldsymbol{\Gamma}}|$ for different directions of unit vectors $\hat{\boldsymbol{\Gamma}}$. We have to define the norm in an appropriate way. Making the time it takes for a sphere with a typical velocity $v_{0}$ to cross the distance of a typical radius of a sphere $a_{0}$ our unit of time (i.e., $a_{0} / v_{0}$ is set to 1) solves the problem that the components of $\hat{\boldsymbol{\Gamma}}$ have different dimensions. For the inner product between two tangent vectors $\delta \boldsymbol{\Gamma}^{(1)}=\left(\delta \mathbf{R}^{(1)}, \delta \mathbf{V}^{(1)}\right)$ and $\delta \boldsymbol{\Gamma}^{(2)}=\left(\delta \mathbf{R}^{(2)}, \delta \mathbf{V}^{(2)}\right)$ we use

$$
\left\langle\delta \boldsymbol{\Gamma}^{(1)} \mid \delta \boldsymbol{\Gamma}^{(2)}\right\rangle=\sum_{i=1}^{N}\left(\delta \mathbf{r}_{i}^{(1)} \cdot \delta \mathbf{r}_{i}^{(2)}+\delta \mathbf{v}_{i}^{(1)} \cdot \delta \mathbf{v}_{i}^{(2)}\right) .
$$

The norm is now defined as $|\delta \boldsymbol{\Gamma}|=\sqrt{\langle\delta \boldsymbol{\Gamma} \mid \delta \boldsymbol{\Gamma}\rangle}$.

The Lyapunov exponents are the logarithms of the eigenvalues of the matrix $\Lambda$, defined by

$$
\Lambda=\lim _{t \rightarrow \infty}\left[\tilde{\mathrm{L}}\left(t-t_{0}\right)\right]^{1 /\left[2\left(t-t_{0}\right)\right]},
$$

where, $\tilde{\mathrm{L}}\left(t-t_{0}\right)=\left[\mathrm{L}\left(t-t_{0}\right)\right]^{\mathrm{T}} \mathrm{L}\left(t-t_{0}\right)$. The corresponding directions for the exponential expansion and contraction of the phase space $(\mathbf{R}, \mathbf{V})$ are obtained from the eigenvectors of $\tilde{\mathrm{L}}\left(t-t_{0}\right)$.

The dynamics of $\delta \boldsymbol{\Gamma}(t)$ in Eq. (2.11), for a gas of hard spheres, can be decomposed into an alternating sequence of flights and instantaneous binary collisions. We denote the transformation of $\delta \boldsymbol{\Gamma}(t)$ over a flight segment between $t$ and $t+\Delta t$ by $\mathrm{H}(\Delta t)$ such that

$$
\delta \boldsymbol{\Gamma}(t+\Delta t)=\mathbf{H}(\Delta t) \delta \boldsymbol{\Gamma}(t) \quad \text { with } \quad \mathbf{H}(0)=\mathrm{I} .
$$

Explicitly, $\mathbf{H}(\Delta t)$ is obtained from

$$
\delta \dot{\boldsymbol{\Gamma}}(t)=\mathbf{T}(t) \delta \boldsymbol{\Gamma}(t)
$$

as

$$
\mathrm{H}(\Delta t)=\exp _{\mathrm{T}}\left[\int_{t}^{t+\Delta t} d t^{\prime} \mathbf{T}\left(t^{\prime}\right)\right],
$$

where the subscript $\mathrm{T}$ indicates time ordering. Notice that $\mathrm{H}(\Delta t)$ in a general system will depend on time $t$ as well, but we have suppressed that in our notation. If we now denote the transformation of $\delta \boldsymbol{\Gamma}(t)$ over an instantaneous binary collision (say, between the $i$-th and the $j$-th sphere) by the matrix $\mathrm{M}_{i j}$, we can express the matrix $\mathrm{L}\left(t-t_{0}\right)$ in terms of the $\mathrm{H}$ and $\mathrm{M}_{i j}$ matrices in the following way: if the dynamics involves flight segments separated by $s$ instantaneous binary collisions at $t_{1}, t_{2} \ldots t_{s}$ such that $t_{0}<t_{1}<t_{2}<\ldots<t_{s}<t$, then

$$
\begin{array}{r}
\mathrm{L}\left(t-t_{0}\right)=\mathrm{H}\left(\Delta t_{s}\right) \mathrm{M}_{i_{s} j_{s}} \mathrm{H}\left(\Delta t_{s-1}\right) \ldots \\
\ldots \mathrm{M}_{i_{1} j_{1}} \mathrm{H}\left(\Delta t_{0}\right) .
\end{array}
$$

Here, $\Delta t_{i}=t_{i+1}-t_{i}$ for $i=1,2, \ldots,(s-1)$ and $\Delta t_{s}=t-t_{s}$. 


\section{The Sufficient Conditions for an Exact CPR}

If the CPR is exactly satisfied for a dynamical system, the sum of the conjugate pairs of the Lyapunov exponents is some constant $c$, i.e., if $\lambda_{i}$ is a Lyapunov exponent of this system, then $c-\lambda_{i}$ is also a Lyapunov exponent. The proof of a possible conjugate pairing rule will follow from the properties of the matrix $\mathrm{L}\left(t-t_{0}\right)$. However, to understand the interplay between the properties of the matrix $\mathrm{L}\left(t-t_{0}\right)$ and an exact CPR in full generality, below we first look at the property of $\mathrm{L}\left(t-t_{0}\right)$ that has been used in various cases to prove CPR.

(a) If the matrix $\mathrm{L}\left(t-t_{0}\right)$ is symplectic, i.e., $\mathrm{L}\left(t-t_{0}\right)$ satisfies the symplectic condition

$$
\left[\mathrm{L}\left(t-t_{0}\right)\right]^{\mathrm{T}} \mathrm{JL}\left(t-t_{0}\right)=\mathrm{J},
$$

with $\mathrm{J}$ as the usual symplectic matrix, then

$$
\tilde{\mathrm{L}}\left(t-t_{0}\right) \mathrm{J} \tilde{\mathrm{L}}\left(t-t_{0}\right)=\mathrm{J} .
$$

Eq. (2.17) can be used to show that

$$
\operatorname{Det}\left[\tilde{\mathrm{L}}\left(t-t_{0}\right)-\tilde{L} \mathrm{I}\right]=0
$$

(with I the identity matrix) implies

$$
\operatorname{Det}\left[\tilde{\mathrm{L}}\left(t-t_{0}\right)-\frac{1}{\tilde{L}} \mathrm{I}\right]=0
$$

This means that if $\tilde{L}$ is an eigenvalue of $\tilde{\mathrm{L}}\left(t-t_{0}\right)$ then so is $\tilde{L}^{-1}$. Since the Lyapunov exponents are the logarithms of the eigenvalues of the matrix $\Lambda$, defined in Eq. 2.12), it is easy to see that $c=0$. All Hamiltonian systems fall in this class.

(b) In the existing literature [8 11], the concept of the symplectic condition defined above, has been generalized to the so-called " $\mu$-symplectic condition" and applied to thermostatted systems where an isokinetic Gaussian thermostat keeps the total laboratory kinetic energy constant and the external force on the constituent particles of the system is dependent only on the positions of the particles. For these systems, in an appropriate reduced phase space characterized by all the non-zero Lyapunov exponents, the matrix $\mathrm{L}\left(t-t_{0}\right)$ satisfies this $\mu$ symplectic condition, which means that there exists a time-dependent positive scalar quantity $\mu$, such that $\left[\mathrm{L}\left(t-t_{0}\right)\right]^{\mathrm{T}} \mathrm{JL}\left(t-t_{0}\right)=\mu \mathrm{J}$. This implies that

$$
\tilde{\mathrm{L}}\left(t-t_{0}\right) \mathrm{J} \tilde{\mathrm{L}}\left(t-t_{0}\right)=\mu^{2} \mathrm{~J}
$$

which can be used to derive that if

$$
\operatorname{Det}\left[\tilde{\mathrm{L}}\left(t-t_{0}\right)-\tilde{L} \mathrm{I}\right]=0
$$

then also

$$
\operatorname{Det}\left[\tilde{\mathrm{L}}\left(t-t_{0}\right)-\frac{\mu^{2}}{\tilde{L}} \mathrm{I}\right]=0
$$

for an eigenvalue $\tilde{L}$ of the matrix $\tilde{L}\left(t-t_{0}\right)$. That means that if $\tilde{L}$ is an eigenvalue of $\tilde{L}\left(t-t_{0}\right)$ then so is $\mu^{2} \tilde{L}^{-1}$. In that case, one finds from Eq. 2.12) that $c=\lim _{t \rightarrow \infty}(\ln \mu) /\left(t-t_{0}\right)$. If the system is ergodic, then this long time average for $c$ can be equated to a NESS average. Notice that condition (a) is obtained as special case of condition (b), namely when $\mu=1$.

Returning momentarily to the SLLOD dynamics, we observe that the formalism developed in Refs. [8 11] fails here. The primary reason is associated with the fact that the total peculiar kinetic energy is held constant for the SLLOD dynamics, as opposed to the total laboratory kinetic energy in Refs. 8 11]. One however needs to interpret the statement regarding the connection between the violation of condition (b) and the non-exactness of the CPR with care. By virtue of the fact that condition (b) above is a sufficient condition for the CPR to hold exactly, the violation of an exact CPR cannot be guaranteed if condition (b) is not satisfied.

Guided by this observation, the interplay between the properties of the matrix $\mathrm{L}\left(t-t_{0}\right)$ and an exact CPR for a dynamical system can be generalized further than what is presented in (b). If there exists any constant nonsingular matrix $\mathrm{K}$ satisfying $\mathrm{K}^{2} \propto \mathrm{I}$ and the following condition

$$
\left[\mathrm{L}\left(t-t_{0}\right)\right]^{\mathrm{T}} \mathrm{KL}\left(t-t_{0}\right)=\mu \mathrm{K}
$$

is satisfied with a time-dependent scalar quantity $\mu$, then Eq. (2.20) can be shown to hold for an eigenvalue $L$ of the matrix $\tilde{L}\left(t-t_{0}\right)$, implying that the CPR is exactly satisfied for such a dynamical system [19]. In analogy with the nomenclature presented in (b), we call Eq. (2.21) a "generalized $\mu$-symplectic condition" with matrix K. We emphasize that the necessary condition for an exact CPR to hold for a dynamical system is not known.

In view of Eq. (2.21), thus, one should look for such a matrix $\mathrm{K}$ to prove an exact CPR. Instead, we look at it from a different angle, namely that we would like to understand how the SLLOD dynamics of $N$ hard spheres with an iso-kinetic Gaussian thermostat deviates from an exact CPR. 


\section{BEHAVIOR OF $\alpha$ IN THE THERMODYNAMIC LIMIT}

Our procedure to study the deviations from CPR begins with the following observation: in the thermodynamic limit, for the SLLOD dynamics with short-range inter-particle potentials at low density of spheres and at small $\gamma$, the behavior of $\alpha$ simplifies to a great extent. After some transient time the system reaches the NESS, and the coefficient of friction $\alpha$ fluctuates with $1 / \sqrt{N}$ fluctuations around a fixed value $\alpha_{0}[16]$. For not too large fluctuations, the distribution function for $\alpha$ can also be shown to be approximately Gaussian. Thus, to calculate the Lyapunov exponents for large $N$ at low density of spheres and at small $\gamma$, to which we confine ourselves henceforth, $\alpha$ can be replaced by $\alpha_{0}$ in Eq. (2.7). We will now briefly present the gist of the derivation in Ref. [16], applied to hard spheres.

For a hard sphere system, the force term in Eqs. (2.1) and (2.2) is zero during a flight. Thus, for a flight, we have

$$
\alpha=-\gamma\left[\sum_{i=1}^{N} p_{i}^{2}\right]^{-1} \sum_{i=1}^{N} p_{i x} p_{i y} .
$$

Introducing a second thermostat variable,

$$
\beta=\gamma^{2}\left[\sum_{i=1}^{N} p_{i}^{2}\right]^{-1} \sum_{i=1}^{N} p_{i y}^{2},
$$

a closed set of equation follows from Eq. (2.1):

$$
\begin{aligned}
& \dot{\alpha}=-2 \alpha^{2}+\beta \\
& \dot{\beta}=-2 \alpha \beta .
\end{aligned}
$$

These equations are valid during the flights, i.e., the intervals between collisions.

We treat collisions by looking at their net effect, i.e., how the velocities and positions of the particles $i$ and $j$ involved in the collision, are changed from their precollisional values $\mathbf{p}_{i-}$ and $\mathbf{p}_{j-}$ to their post-collisional values $\mathbf{p}_{i+}$ and $\mathbf{p}_{j+}$. These are the only two velocities to change, and because $\sum_{i=1}^{N} p_{i}^{2}$ is of order $N$, the changes in $\alpha$ and $\beta$ are, according to Eqs. (3.2) and (3.3), of order $N^{-1}$. The number of collisions in the whole system is an extensive quantity as well, so there are $O(N)$ of these $O(1 / N)$ changes in a unit of time. The averages of the small changes are not zero, so there is a net effect of $O(1)$ per unit time to the time derivatives of $\alpha$ and $\beta$, which we will denote by $a$ respectively, $b$ :

$$
\begin{aligned}
& \dot{\alpha}=-2 \alpha^{2}+\beta+a \\
& \dot{\beta}=-2 \alpha \beta+b .
\end{aligned}
$$

This set of equations has a fixed point $\left(\alpha_{0}, \beta_{0}\right)$, which is stable if $\alpha_{0}>0$, so the system reaches this fixed point after some time. On top of this dynamics, there are fluctuations. Assuming that the collisions are independent, the central limit theorem applies, and the fluctuations are $O(1 / \sqrt{N})$. For more detailed analysis, we refer to Ref. [16], where the independence of the changes in $\alpha$ and $\beta$ is linked to the assumption of molecular chaos.

Finally, we note that to maintain a stationary total peculiar kinetic energy in a system with a constant $\alpha_{0}$ thermostat, this constant has to be chosen differently for varying $\gamma$. For $\gamma=0$, i.e., in equilibrium, $\alpha_{0}$ can be set to zero, and kinetic energy is determined by the initial conditions. Near equilibrium, i.e., in the linear response regime, the right hand side of Eq. (3.1) should scale as $\gamma^{2}$, i.e. $\alpha_{0} \propto \gamma^{2}$. Obviously, there are higher order corrections to this behavior which play a role for larger values of $\gamma$. If, for $\gamma \neq 0$, the initial condition is such that the total peculiar kinetic energy is not equal to the stationary value, that value will be approached in time.

\section{STATUS OF AN APPROXIMATE CPR IN THE THERMODYNAMIC LIMIT}

Based on the discussion in the last paragraph of Sec. II and using the results in Sec. III, we will explore the possibility of an approximate CPR for the the SLLOD dynamics of hard spheres in the thermodynamic limit, at small $\gamma$ and at low density in this section. We will first obtain the desired results for a constant coefficient of friction $\alpha_{0}$ in the equations of motion (2.7) and then we will discuss the validity of an approximate CPR when the system is under an iso-kinetic Gaussian thermostat. To this end, our starting aim is to study the generalized $\mu$-symplectic properties of the matrix $\mathrm{L}\left(t-t_{0}\right)$ for the dynamics described by Eq. (2.7) during a flight and Eq. (2.9) during an instantaneous binary collision between the $i$-th and the $j$-th sphere.

However, as the matrix $\mathrm{L}\left(t-t_{0}\right)$ is constructed from the $\mathrm{H}$ and the $\mathrm{M}$ matrices, we will have to study the generalized $\mu$-symplectic properties of the $\mathrm{H}$ and the $\mathrm{M}$ matrices separately.

\section{A. Generalized $\mu$-symplecticity Property of $\mathrm{H}(\Delta t)$}

The matrix $\mathrm{T}$ describing the dynamics of $\delta \boldsymbol{\Gamma}$ during flights is found from Eq. (2.7) to be

$$
\mathbf{T} \equiv\left[\begin{array}{cc}
0 & \mathrm{I} \\
\alpha_{0} \gamma \mathrm{C} & -\alpha_{0} \mathrm{I}
\end{array}\right] \text {. }
$$

From Eq. (4.1), it is straightforward to obtain

$$
\mathrm{H}(\Delta t)=\exp [\mathrm{T} \Delta t],
$$

where the $3 \times 3$ sub-blocks of $\mathrm{H}$ are given by $\mathrm{H}_{l m}^{[k]}(\Delta t)=$ $\mathrm{h}^{(k)}(\Delta t) \delta_{l, m}$, where 


$$
\begin{aligned}
\mathrm{h}^{(1)}(\Delta t)=\mathrm{I} & +\left[\gamma \Delta t-\frac{\gamma\left[1-\exp \left(-\alpha_{0} \Delta t\right)\right]}{\alpha_{0}}\right] \mathrm{c}, \\
\mathrm{h}^{(2)}(\Delta t)= & \frac{1-e^{-\alpha_{0} \Delta t}}{\alpha_{0}} \mathrm{I} \\
& +\frac{\gamma}{\alpha_{0}^{2}}\left[\alpha_{0} \Delta t\left(1+e^{-\alpha_{0} \Delta t}\right)-2+2 e^{-\alpha_{0} \Delta t}\right] \mathrm{c}, \\
\mathrm{h}^{(3)}(\Delta t)= & \gamma\left[1-e^{-\alpha_{0} \Delta t}\right] \mathrm{c} \text { and } \\
\mathrm{h}^{(4)}(\Delta t)= & e^{-\alpha_{0} \Delta t}\left\{\mathrm{I}-\gamma\left[\Delta t+\frac{1}{\alpha_{0}}\left[1-e^{\alpha_{0} \Delta t}\right]\right] \mathrm{c}\right\} .
\end{aligned}
$$

Due to the complicated form of $\mathrm{H}(\Delta t)$, it is easier to study its $\mu$-symplecticity properties in terms of the matrix T. This involves the task of finding a possible matrix $\mathrm{K}$ satisfying the condition

$$
\mathrm{T}^{\mathrm{T}} \mathrm{K}+\mathrm{KT}=\beta \mathrm{K}
$$

such that $\mathrm{K}^{2} \propto \mathrm{I}$. If such a matrix $\mathrm{K}$ exists, then $\mathrm{H}(\Delta t)$ is generalized $\mu$ symplectic with that matrix, and

$$
\mu=\exp \left[\int_{t}^{t+\Delta t} \beta d t^{\prime}\right] .
$$

Since $\mathrm{K}$ and $\mathrm{T}$ are constant matrices in the present context, $\beta$ is also a constant. Eq. (4.4) can be treated as a simple eigenvalue equation to solve for the eigenvalue $\beta$ and the eigenvector $\mathrm{K}$. We find that there exists a matrix G satisfying

$$
\mathrm{T}^{\mathrm{T}} \mathrm{G}+\mathrm{GT}=-\alpha_{0} \mathrm{G}
$$

and the $6 N \times 6 N$ matrix $\mathrm{G}$ in terms of its $3 \times 3$ sub-blocks, is given by $\mathrm{G}^{[1]}=\mathrm{G}^{[4]}=0$ and $\mathrm{G}_{l m}^{[2]}=-\mathrm{G}_{l m}^{[3]}=\mathrm{g}$, where

$$
\mathrm{g}=\left[\begin{array}{lll}
0 & 1 & 0 \\
1 & 0 & 0 \\
0 & 0 & 1
\end{array}\right]
$$

We note that there may exist other forms of $g$ such that G satisfies Eq. (4.6), but Eq. (4.7) is the simplest one that satisfies $\mathrm{G}^{2} \propto \mathrm{I}$ and works for all $\gamma$.

For the purpose of future use, we construct matrices $\mathrm{T}_{0}$ and $\mathrm{H}_{0}(\Delta t)$ by setting $\gamma=0$ in Eq. (4.1) and Eq. (4.3), without setting $\alpha_{0}=0$ (in reality, $\alpha_{0}=0$ when $\gamma=0$ ), i.e.,

$$
\begin{aligned}
\mathrm{T}_{0} & \equiv\left[\begin{array}{cc}
0 & \mathrm{I} \\
0 & -\alpha_{0} \mathrm{I}
\end{array}\right] \text { and } \\
\mathrm{H}_{0}(\Delta t) & \equiv \exp \left[\mathrm{T}_{0} \Delta t\right] .
\end{aligned}
$$

More explicitly, the $3 \times 3$ sub-blocks of $\mathrm{H}_{0}(\Delta t)$ are given by $\left(\mathrm{H}_{0}\right)_{l m}^{[k]}(\Delta t)=\mathrm{h}_{0}^{(k)}(\Delta t) \delta_{l, m}$, and $\mathrm{h}_{0}^{(k)}(\Delta t)$ can be found by putting $\gamma=0$ in Eq. (4.3) without putting $\alpha_{0}=0$. The matrix $\mathrm{H}_{0}(\Delta t)$ is now not only generalized $\mu$-symplectic with matrix $\mathrm{G}$, but also $\mu$-symplectic with J, i.e.,

$$
\left[\mathrm{H}_{0}(\Delta t)\right]^{\mathrm{T}} \mathrm{J} \mathrm{H}_{0}(\Delta t)=e^{-\alpha_{0} \Delta t} \mathrm{~J}
$$

The relevance of this observation will become clear in Sec. IV.C.

We also note that Eqs. (4.1-4.7) hold for any constant coefficient of friction (not necessarily $\alpha_{0}$ ), which implies, using Eq. (2.21), that for a collisionless gas of point particles obeying the SLLOD dynamics with a constant coefficient of friction, the CPR is exact, as can be seen in simulations 13.

\section{B. Generalized $\mu$-symplecticity Property of $\mathrm{M}_{i j}$}

Unlike the $\mathrm{H}$ matrices, the $\mathrm{M}_{i j}$ matrices corresponding to a binary collision between the $i$-th and the $j$-th sphere do not follow from Eq. (2.9) directly. This is due to the fact that even though the sequence of binary collisions are the same on the reference and the adjacent trajectories, the binary collision between the $i$-th and the $j$-th sphere on these two trajectories in the phase space are not simultaneous. One therefore needs the dynamics of the tangent vectors for the time interval, $\delta \tau$, between the two collisions on the reference and the adjacent trajectories involving the $i$-th and the $j$-th sphere. To obtain an expression of $\mathrm{M}_{i j}$, we follow the formalism developed in Refs. 11, 22, which in turn, is based on the formalism presented in Refs. [18 and 20].

The dynamics of tangent vectors at a collision is derived in the Appendix. The result is that

$$
\delta \boldsymbol{\Gamma}_{+}=\mathrm{M}_{i j} \delta \boldsymbol{\Gamma}_{-}
$$

with

$$
\mathrm{M}_{i j}=\left(\mathrm{I}-2 \hat{\mathbf{N}}_{i j} \hat{\mathbf{N}}_{i j}\right)\left[\begin{array}{ll}
\mathrm{I} & 0 \\
\mathrm{R} & \mathrm{I}
\end{array}\right],
$$

and $\mathrm{R}$ a symmetric matrix given by Eq. (A18). This form of $\mathrm{M}_{i j}$ immediately implies that $\mathrm{M}$ is symplectic, but not generalized $\mu$-symplectic with matrix $\mathrm{G}$ for $\mu=1$, i.e.,

$$
\mathrm{M}_{i j}^{\mathrm{T}} \mathrm{JM}_{i j}=\mathrm{J}
$$

but

$$
\mathrm{M}_{i j}^{\mathrm{T}} \mathrm{GM}_{i j} \neq \mathrm{G}
$$

\section{Generalized $\mu$-symplecticity Property of $\mathrm{L}\left(t-t_{0}\right)$ and the Origin of an Approximate CPR}

From Secs. IV.A and IV.B above, we can finally see that for a collection of hard spheres obeying the SLLOD equations of motion with constant coefficient of friction $\alpha_{0}$

(a) the $\mathrm{H}$ matrices are generalized $\mu$-symplectic with matrix G, but not with matrix J (see Eq. (4.6) 
(b) the $\mathrm{M}$ matrices are symplectic but not generalized $\mu$-symplectic with matrix G (see Eqs. (4.13) and (4.14)) .

Once the $\mathrm{H}$ and the $\mathrm{M}$ matrices are combined together, following Eq. (2.16), the matrix

$\mathrm{L}\left(t-t_{0}\right)=\mathrm{H}\left(\Delta t_{s}\right) \mathrm{M}_{i_{s} j_{s}} \mathrm{H}\left(\Delta t_{s-1}\right) \ldots \mathrm{M}_{i_{1} j_{1}} \mathrm{H}\left(\Delta t_{0}\right)$

is seen to be generalized $\mu$-symplectic neither with matrix $\mathrm{G}$ nor with matrix J.

To study the degree of deviation from an exact CPR using the properties of $\mathrm{L}\left(t-t_{0}\right)$ in Eq. 4.15) with constant coefficient of friction $\alpha_{0}$, we can use either $\mathrm{K}=\mathrm{G}$ or $\mathrm{K}=\mathrm{J}$. While the former choice implies that one has to try to estimate the deviation from an exact CPR using the distribution of the unit vectors $\hat{\mathbf{N}}_{i j}$ 's and the collision angles for different sets of binary collisions in the expression of $\mathrm{M}$, the latter choice means that one can make the estimate by using the property of the $\mathrm{H}$ matrices in Eq. (4.15). We choose the latter approach, because not only is it much easier to calculate the typical magnitude of a flight time of a sphere at low densities, but also, an estimate of the deviation from the exact CPR can be made at small $\gamma$, as a power series expansion in $\gamma$. However, the smallness of $\gamma$, which has a dimension of inverse time, has to be defined in a proper manner. To do so, we notice that the density of the spheres $n$ sets a time scale in the form of the mean flight time $\tau_{0}$ of an individual sphere, and in three dimensions $\tau_{0} \sim \tilde{n} a_{0} / v_{0}$. Here, $\tilde{n}=n a_{0}^{3}$ is the dimensionless density and, as before, $v_{0}$ and $a_{0}$ are typical velocity and radius of a sphere. Thus, the actual dimensionless small parameter corresponding to the shear rate is $\tilde{\gamma}=\gamma \tau_{0}$.

A naive way to estimate the deviation from an exact CPR using the latter approach is to use the deviation of the $\mathrm{H}(\Delta t)$ matrices from an exact $\mu$-symplecticity (see Eq. (4.15)). Such a deviation is characterized by the matrix $\mathrm{D}(\Delta \mathrm{t})=[\mathrm{H}(\Delta t)]^{\mathrm{T}} \mathrm{JH}(\Delta t)-e^{-\alpha_{0} \Delta t} \mathrm{~J}$. The matrix $\mathrm{D}(\Delta t)$ can easily be calculated from Eq. (4.3). However, to estimate the order of the matrix elements of $\mathrm{D}(\Delta t)$, an order of estimate of the quantity $\Delta t$ has to be obtained. To this end, we note that while $\tau_{0}$ is the mean flight time for an individual sphere, $\Delta t$ in Eq. (4.15) denotes the mean time for a flight of $N$ spheres. This implies that $\Delta t \sim \tau_{0} / N$, as on an average, there are $N / 2$ different binary collisions over a mean flight time $\tau_{0}$ of an individual sphere. Thus, one would expect that in the thermodynamic limit, $\mathrm{D}(\Delta t) \rightarrow \mathrm{D}(0)=0$ and one would be led to conclude that the $\mathrm{H}$ matrices in Eq. (4.15) are all symplectic. This in turn would imply, from Eq. (4.15), that $\mathrm{L}\left(t-t_{0}\right)$ would be $\mu$-symplectic and therefore a gas of hard spheres, obeying the SLLOD dynamics with a constant coefficient of friction $\alpha_{0}$ would satisfy an exact CPR in the thermodynamic limit. We demonstrate below that this simplification is not correct.

The proper estimate of the deviation from an exact CPR has to be made by considering $\mathbf{H}\left(\tau_{0}\right)$. To see why this is so, we rewrite the matrix $\mathbf{H}(\Delta t)$ as

$$
\mathbf{H}(\Delta t)=e^{\frac{N-1}{2} \alpha_{0} \Delta t} \prod_{i=1}^{N} \mathbf{H}^{i}(\Delta t)
$$

with $\mathrm{H}^{i}(\Delta t)$ defined by

$$
\begin{aligned}
\left(\mathrm{H}^{i}\right)_{l m}^{[k]}(\Delta t)= & \delta_{l, m}\left[\delta_{l, i} \mathrm{~h}^{(k)}(\Delta t)\right. \\
& \left.+\left(1-\delta_{l, i}\right)\left(\delta_{k, 1}+\delta_{k, 4}\right) e^{-\alpha_{0} \Delta t / 2} \mathbf{I}\right] .
\end{aligned}
$$

In effect, $\mathrm{H}^{i}(\Delta t)$ describes the evolution of the infinitesimal deviation of the trajectory of the $i$-th sphere, while it has an almost trivial action on the infinitesimal deviation of the trajectory of the $j$-th sphere, $j \neq i$. It is easy to see that $\mathrm{H}^{i}(\Delta t)$ has some useful properties

$$
\begin{aligned}
\mathrm{H}^{i}\left(\Delta t_{1}\right) \mathrm{H}^{i}\left(\Delta t_{2}\right) & =\mathrm{H}^{i}\left(\Delta t_{1}+\Delta t_{2}\right), \\
{\left[\mathrm{H}^{i}\left(\Delta t_{i}\right), \mathrm{H}^{j}\left(\Delta t_{j}\right)\right] } & =0 \quad \text { and } \\
{\left[\mathrm{H}^{i}(\Delta t), \mathrm{M}_{i_{c} j_{c}}\right] } & =0 \quad \text { if } i_{c} \neq i \text { and } j_{c} \neq i .
\end{aligned}
$$

The properties of $\mathrm{H}^{i}(\Delta t)$ in Eq. (4.18) allow us to shuffle the terms in Eq. (4.15) such as to collect together as many $\mathrm{H}^{i}(\Delta t)$ s with the same $i$ as possible. The result is that to the right of any $\mathbf{M}_{i_{c} j_{c}}$ figure an $\mathbf{H}^{i_{c}}\left(\tau_{i_{c}}^{c}\right)$ and an $\mathrm{H}^{j_{c}}\left(\tau_{j_{c}}^{c}\right)$, where $\tau_{i_{c}}^{c}$ and $\tau_{j_{c}}^{c}$ are the time of flights for the $i_{c}$-th and the $j_{c}$-th spheres before their mutual collision c. Consequently,

$$
\begin{aligned}
\mathrm{L}\left(t-t_{0}\right)= & e^{\frac{N-1}{2} \alpha_{0}\left(t-t_{0}\right)}\left\{\prod_{i=1}^{N} \mathrm{H}^{i}\left(t-\tilde{t}_{i}\right)\right\} \\
& \times \prod_{c=1}^{s} \mathrm{M}_{i_{c} j_{c}} \mathrm{H}^{i_{c}}\left(\tau_{i_{c}}^{c}\right) \mathrm{H}^{j_{c}}\left(\tau_{j_{c}}^{c}\right) .
\end{aligned}
$$

The product sign in Eq. (4.19) is to be expanded towards the left, i.e. $\prod_{c=1}^{s} A_{c}=A_{s} \cdots A_{1}$. Here, $\tilde{t}_{i}$ is the last time that $i$-th particle collided (or $t_{0}$ if it didn't collide). From Eq. (4.19), it is now clear that the proper estimate for the deviation from an exact CPR has to be made by considering the properties of $\mathrm{H}^{i}\left(\tau_{i}\right)$, with $\tau_{i}=O\left(\tau_{0}\right)$, and not from the properties of $\mathrm{H}^{i}\left(\tau_{0} / N\right)$.

We also notice that if one uses the corresponding $\mathrm{H}_{0}$ matrices instead of the $\mathrm{H}$ matrices in Eq. (4.15) to construct an analogous matrix $\mathrm{L}_{0}\left(t-t_{0}\right)$, defined by

$$
\begin{aligned}
\mathrm{L}_{0}\left(t-t_{0}\right)= & \mathrm{H}_{0}\left(\Delta t_{s}\right) \mathrm{M}_{i_{s} j_{s}} \mathrm{H}_{0}\left(\Delta t_{s-1}\right) \ldots \\
& \ldots \mathrm{M}_{i_{1} j_{1}} \mathrm{H}_{0}\left(\Delta t_{0}\right),
\end{aligned}
$$

then the matrix $\mathrm{L}_{0}\left(t-t_{0}\right)$ is $\mu$-symplectic, because of Eqs. (4.10) and (4.13). As a consequence, the logarithms of the eigenvalues of $\tilde{L}_{0}\left(t-t_{0}\right)$, defined by

$$
\tilde{\mathrm{L}}_{0}\left(t-t_{0}\right)=\left[\mathrm{L}_{0}\left(t-t_{0}\right)\right]^{\mathrm{T}} \mathrm{L}_{0}\left(t-t_{0}\right),
$$

pair exactly. If we arrange the corresponding Lyapunov spectrum 


$$
\Lambda_{0}=\lim _{t \rightarrow \infty}\left[\tilde{\mathrm{L}}_{0}\left(t-t_{0}\right)\right]^{1 / 2\left(t-t_{0}\right)}
$$

in the decreasing order of magnitude as $\lambda_{1}^{(0)}, \lambda_{2}^{(0)}$, $\ldots, \lambda_{6 N}^{(0)}$, then $\lambda_{i}^{(0)}+\lambda_{6 N-i+1}^{(0)}=-\alpha_{0}$.

Motivated by this, our approach to study the deviation from an exact CPR for the matrix $\mathrm{L}\left(t-t_{0}\right)$ will be to take $\mathrm{L}_{0}\left(t-t_{0}\right)$ as the reference matrix. This is reasonable because, as we will show, $\mathrm{L}\left(t-t_{0}\right)$ and $\mathrm{L}_{0}\left(t-t_{0}\right)$ are very close for small $\tilde{\gamma}$, if $t-t_{0}=\tau=O\left(\tau_{0}\right)$. To show this, we first write the matrix $\mathrm{L}_{0}\left(t-t_{0}\right)$ in the same form as Eq. 4.19), with $\mathrm{H}^{i}\left(\tau_{i}\right) \mathrm{s}$ replaced by $\mathrm{H}_{0}^{i}\left(\tau_{i}\right) \mathrm{s}$. We then relate, for $t-t_{0}=\tau=O\left(\tau_{0}\right)$, the difference matrix $\Delta \mathrm{L}\left(t-t_{0}\right)=\mathrm{L}\left(t-t_{0}\right)-\mathrm{L}_{0}\left(t-t_{0}\right)$ to the difference between $\mathrm{H}^{i}\left(\tau_{i}\right)$ and $\mathrm{H}_{0}^{i}\left(\tau_{i}\right)$.

We now define new matrices $\Delta \mathrm{H}^{i}$ and $\mathrm{E}^{i}$, such that

$$
\begin{aligned}
\Delta \mathrm{H}^{i}\left(\tau_{i}\right) & \equiv \mathrm{H}^{i}\left(\tau_{i}\right)-\mathrm{H}_{0}^{i}\left(\tau_{i}\right) \\
\mathrm{E}^{i}\left(\tau_{i}\right) & \equiv\left[\mathrm{H}_{0}^{i}\left(\tau_{i}\right)\right]^{-1} \Delta \mathrm{H}^{i}\left(\tau_{i}\right)
\end{aligned}
$$

with

$$
\begin{aligned}
\left(\mathrm{H}_{0}^{i}\right)_{l m}^{[k]}\left(\tau_{i}\right)= & \delta_{l, m}\left[\delta_{l, i} \mathrm{~h}_{0}^{(k)}\left(\tau_{i}\right)\right. \\
& \left.+\left(1-\delta_{l, i}\right)\left(\delta_{k, 1}+\delta_{k, 4}\right) e^{-\alpha_{0} \tau_{i} / 2} \mathbf{I}\right]
\end{aligned}
$$

[for the definition of $\mathrm{h}_{0}^{(k)}\left(\tau_{i}\right)$, see the preceding paragraph of Eq. (4.10)]. It is easily seen that $\mathrm{E}^{i}\left(\tau_{i}\right)$ has non-zero elements only for entries involving the $i$-th sphere. This, together with relations 4.18), implies that

$$
\begin{aligned}
& {\left[\mathrm{E}^{i}\left(\tau_{i}\right), \mathrm{H}_{0}^{j}\left(\tau_{j}\right)\right]=0 \text { for } i \neq j \text { and }} \\
& {\left[\mathrm{E}^{i}\left(\tau_{i}\right), \mathrm{M}_{i_{c} j_{c}}\right]=0 \text { if } i_{c} \neq i \text { and } j_{c} \neq i \text {. }}
\end{aligned}
$$

The matrix $E^{i}\left(\tau_{i}\right)$ can be easily calculated from Eqs. (4.23). With the aid of Eqs. (4.3) and (4.9), we now express the matrix $\Delta \mathrm{H}^{i}\left(\tau_{i}\right)$ defined in Eq. (4.23) as $\Delta \mathrm{H}_{l m}^{i(k)}\left(\tau_{i}\right)=\Delta \mathrm{h}^{i[k]}\left(\tau_{i}\right) \delta_{l, m}$, where

$$
\begin{aligned}
\Delta \mathrm{h}^{i(1)}\left(\tau_{i}\right) & =\left[\gamma \tau_{i}-\frac{\gamma\left[1-\exp \left(-\alpha_{0} \tau_{i}\right)\right]}{\alpha_{0}}\right] \mathrm{c}, \\
\Delta \mathrm{h}^{i(2)}\left(\tau_{i}\right) & =\frac{\gamma}{\alpha_{0}^{2}}\left[\alpha_{0} \tau_{i}\left(1+e^{-\alpha_{0} \tau_{i}}\right)-2+2 e^{-\alpha_{0} \tau_{i}}\right] \mathrm{c} \\
\Delta \mathrm{h}^{i(3)}\left(\tau_{i}\right) & =\gamma\left[1-e^{-\alpha_{0} \tau_{i}}\right] \mathrm{c} \text { and } \\
\Delta \mathrm{h}^{i(4)}\left(\tau_{i}\right) & =-\gamma e^{-\alpha_{0} \tau_{i}}\left[\tau_{i}+\frac{1}{\alpha_{0}}\left[1-e^{\alpha_{0} \tau_{i}}\right]\right] \mathrm{c} .
\end{aligned}
$$

The crucial point is that since $\alpha_{0} \propto \gamma \tilde{\gamma}$, i.e., the dissipation is quadratic in the shear field, $\Delta \mathrm{H}^{i}\left(\tau_{i}\right)$ in Eq. (4.26) can be expanded in powers of $\gamma$ to obtain

$$
\begin{aligned}
\Delta \mathrm{h}^{i(1)}\left(\tau_{i}\right) & =\frac{\gamma \alpha_{0} \tau_{i}^{2}}{2 !} \mathrm{c}, \\
\Delta \mathrm{h}^{i(2)}\left(\tau_{i}\right) & =\frac{\gamma \alpha_{0} \tau_{i}^{3}}{3 !} \mathrm{c}, \\
\Delta \mathrm{h}^{i(3)}\left(\tau_{i}\right) & =\gamma \alpha_{0} \tau_{i} \mathrm{c} \text { and } \\
\Delta \mathrm{h}^{i(4)}\left(\tau_{i}\right) & =\frac{\gamma \alpha_{0} \tau_{i}^{2}}{2 !} \mathrm{c}
\end{aligned}
$$

at the most leading order. Thereafter, having combined Eqs. (4.23) and (4.26 4.27), all elements of $\mathrm{E}^{i}\left(\tau_{i}\right)$ are seen to have a prefactor of order $\tilde{\gamma}^{3}$. This makes $\mathrm{E}^{i}\left(\tau_{i}\right)$ small compared to 1 . Hence, to first order, $\Delta \mathrm{L}(\tau)=$ $\mathrm{L}(\tau)-\mathrm{L}_{0}(\tau)$ can be found from Eq. (4.19) by keeping only the terms linear in $\mathrm{E}^{i}\left(\tau_{i}\right)$ :

$$
\begin{aligned}
\Delta \mathrm{L}(\tau)= & e^{\frac{N-1}{2} \alpha_{0} \tau}\left\{\prod_{i=1}^{N} \mathrm{H}_{0}^{i}\left(\tau+t_{0}-\tilde{t}_{i}\right)\right\}\left(\sum_{j=1}^{N} \mathrm{E}^{j}\left(\tau+t_{0}-\tilde{t}_{j}\right) \prod_{c=1}^{s} \mathrm{M}_{c} \mathrm{H}_{0}^{i_{c}}\left(\tau_{i_{c}}^{c}\right) \mathrm{H}_{0}^{j_{c}}\left(\tau_{j_{c}}^{c}\right)\right. \\
& \left.+\sum_{c=1}^{s} \prod_{a=1}^{c} \mathrm{M}_{a} \mathrm{H}_{0}^{i_{a}}\left(\tau_{i_{a}}^{a}\right) \mathrm{H}_{0}^{j_{a}}\left(\tau_{j_{a}}^{a}\right) \times\left[\mathrm{E}^{j_{c}}\left(\tau_{j_{c}}^{c}\right)+\mathrm{E}^{i_{c}}\left(\tau_{i_{c}}^{c}\right)\right] \prod_{b=c+1}^{s} \mathrm{M}_{b} \mathrm{H}_{0}^{i_{b}}\left(\tau_{i_{b}}^{b}\right) \mathrm{H}_{0}^{j_{b}}\left(\tau_{j_{b}}^{b}\right)\right) .
\end{aligned}
$$

We now shuffle all the $\mathrm{E}^{i_{c}}$ matrices to the right, by successively exchanging the order of $\mathrm{E}^{i_{c}}$ and the next $\mathrm{M}_{b} \mathrm{H}_{0}^{i_{b}}\left(\tau_{i_{b}}^{b}\right) \mathrm{H}_{0}^{j_{b}}\left(\tau_{j_{b}}^{b}\right)$ to its right. These will commute very often, because most collisions in the sequence will involve other spheres than this $i_{c}$-th one. If they do not commute, we write

$$
\mathrm{E}^{i_{c}} \mathrm{M}_{b} \mathrm{H}_{0}^{i_{b}}\left(\tau_{i_{b}}^{b}\right) \mathrm{H}_{0}^{j_{b}}\left(\tau_{j_{b}}^{b}\right)=\mathrm{M}_{b} \mathrm{H}_{0}^{i_{b}}\left(\tau_{i_{b}}^{b}\right) \mathrm{H}_{0}^{j_{b}}\left(\tau_{j_{b}}^{b}\right)\left[\mathrm{M}_{b} \mathrm{H}_{0}^{i_{b}}\left(\tau_{i_{b}}^{b}\right) \mathrm{H}_{0}^{j_{b}}\left(\tau_{j_{b}}^{b}\right)\right]^{-1} \mathrm{E}^{i} \mathrm{M}_{b} \mathrm{H}_{0}^{i_{b}}\left(\tau_{i_{b}}^{b}\right) \mathrm{H}_{0}^{j_{b}}\left(\tau_{j_{b}}^{b}\right) .
$$

From that point on, we work with " $\left[\mathrm{M}_{b} \mathrm{H}_{0}^{i_{b}} \mathrm{H}_{0}^{j_{b}}\right]^{-1} \mathrm{E}^{i} \mathrm{M}_{b} \mathrm{H}_{0}^{i_{b}} \mathrm{H}_{0}^{j_{b}}$ ", and shuffle that to the right. Repeating this, we end up with $\mathrm{L}_{0}(\tau)$ on the left side again, and find, symbolically,

$$
\begin{aligned}
{\left[\mathrm{L}_{0}(\tau)\right]^{-1} \Delta \mathrm{L}(\tau)=} & \sum_{c=1}^{s}\left\{\prod\left(\mathrm{MH}_{0} \mathrm{H}_{0}\right)^{-1}\right\}\left[\mathrm{E}^{j_{c}}\left(\tau_{j_{c}}^{c}\right)+\mathrm{E}^{i_{c}}\left(\tau_{i_{c}}^{c}\right)\right]\left\{\prod \mathrm{MH}_{0} \mathrm{H}_{0}\right\} \\
& +\sum_{j=1}^{N}\left\{\prod\left(\mathrm{MH}_{0} \mathrm{H}_{0}\right)^{-1}\right\} \mathrm{E}^{j}\left(\tau+t_{0}-\tilde{t}_{j}\right)\left\{\prod \mathrm{MH}_{0} \mathrm{H}_{0}\right\} .
\end{aligned}
$$


Since $\tau$ is of the order of a flight time of a sphere, $s$ is of the order of $N$, but a typical sphere will have suffered only a few [a few here is roughly $O(1)$ ] collisions. Consider a typical term in the sum over $c$. The $\mathrm{MH}_{0} \mathrm{H}_{0}$ terms in the products are from collisions that involve the $i_{c}$-th or the $j_{c}$-th sphere, or spheres that were involved in collisions with the $i_{c}$-th or $j_{c}$-th sphere. The number of $\mathrm{MH}_{0} \mathrm{H}_{0}$ will thus also be of $O(1)$.

The matrix $E^{i_{c}}$ has non-zero elements only for the entries associated with the $i_{c}$-th sphere. The multiplication by $\mathrm{MH}_{0} \mathrm{H}_{0}$ yields more non-zero entries associated with other spheres, whose number however is of $O(1)$. Therefore, in the whole sum of $s=O(N)$ terms in Eq. (4.30) will yield for typical entries a result of order one (and not of order $N$ as the sum over $s \propto N$ suggests). Since all elements of the $\mathrm{E}$ matrices are proportional to $\gamma \tilde{\gamma}^{2}$ as can be seen from Eqs. (4.23), (4.26) and (4.27), we conclude for $t-t_{0}=\tau=O\left(\tau_{0}\right)$ that

$$
\mathrm{L}(\tau)=\mathrm{L}_{0}(\tau)\left[\mathrm{I}+\tilde{\gamma}^{3} \mathrm{~B}\right],
$$

where the matrix $\mathrm{B}$ is of order 1 in $\tilde{\gamma}$ and order 1 in $N$. Note that B contains higher powers of $\tilde{\gamma}$ as well. Because it involves $\mathrm{C}$ and contributions from collisions between spheres, B is not proportional to the identity I, so we cannot regard it as a simple scalar factor (in which case the exact conjugate pairing would be easy to obtain again). Equation (4.31) implies

$$
\begin{aligned}
\Delta \tilde{\mathrm{L}}(\tau) & =\tilde{\mathrm{L}}(\tau)-\tilde{\mathrm{L}}_{0}(\tau) \\
& =\tilde{\gamma}^{3}\left[\mathrm{~B}^{\mathrm{T}} \tilde{\mathrm{L}}_{0}(\tau)+\tilde{\mathrm{L}}_{0}(\tau) \mathrm{B}\right]+\tilde{\gamma}^{6} \mathrm{~B}^{\mathrm{T}} \tilde{\mathrm{L}}_{0}(\tau) \mathrm{B} .
\end{aligned}
$$

We can now see, from Eqs. (4.31) and (4.32) that the differences between $\mathrm{L}(\tau)$ and $\mathrm{L}_{0}(\tau)$, and between $\tilde{\mathrm{L}}(\tau)$ and $\tilde{\mathrm{L}}_{0}(\tau)$ are small, as anticipated on page 8 , by a relative order $\tilde{\gamma}^{3}$. Therefore the logarithm of the eigenvalues of $\mathrm{L}(\tau)$ and $\mathrm{L}_{0}(\tau)$ also differ by a term of order $\tilde{\gamma}^{3}$ in an absolute sense. If we now divide the logarithms of these eigenvalues by the time $\tau$, we see that the finite time (for time $\tau$ ) Lyapunov exponents, calculated from $\tilde{\mathrm{L}}_{0}$ and from $\tilde{\mathrm{L}}$ (which we denote as $\lambda_{i}^{(0)}(\tau)$ and $\lambda_{i}(\tau)$ respectively, for $i=1,2 \ldots 6 N)$ differ by a term $O\left(\tilde{\gamma}^{3} / \tau\right)=O\left(\gamma \tilde{\gamma}^{2}\right)$. Using the fact that that $\lambda_{i}^{(0)}(\tau)$ s satisfy the conjugate pairing rule, $\lambda_{i}(\tau)$ s too, will satisfy it up to $O\left(\gamma \tilde{\gamma}^{2}\right)$. From the condition that

$$
\sum_{i=1}^{6 N} \lambda_{i}^{(0)}(\tau)=\sum_{i=1}^{6 N} \lambda_{i}(\tau)=-3 N \alpha_{0},
$$

[which can be easily verified from Eqs. (4.3) and (4.12)], we see that on average over the pairs, the deviation from CPR is zero.

We make one further observation at this stage. The Lyapunov exponents (even the finite time once) are invariant under $\gamma \rightarrow-\gamma$, so in a power series expansion of the individual Lyapunov exponents in $\tilde{\gamma}$ 21, the odd powers vanish. We therefore conclude that the logarithm of the eigenvalues of $\mathrm{L}(\tau)$ and $\mathrm{L}_{0}(\tau)$ must differ by a term of order $\tilde{\gamma}^{4}$, and hence conjugate pairing of $\lambda_{i}(\tau)$ s will be valid up to the correction of the form $\gamma \tilde{\gamma}^{3}$.

To extend these ideas for large $\left(t-t_{0}\right)$ [and finally for $t \rightarrow \infty)$ ], we proceed in the following way. Notice that the matrices $\tilde{\mathrm{L}}\left(t-t_{0}\right)$ and $\tilde{\mathrm{L}}_{0}\left(t-t_{0}\right)$ are positive definite and symmetric. This allows us to express the two matrices in the form of $\tilde{\mathrm{L}}_{0}\left(t-t_{0}\right)=\exp \left(\mathrm{A}_{0}\right)$ and $\tilde{\mathrm{L}}\left(t-t_{0}\right)=\exp (\mathrm{A})$, where for large $\left(t-t_{0}\right)$, both the eigenvalues of $\mathrm{A}_{0}$ and A must behave $\sim\left(t-t_{0}\right)$. In these terms, the difference between the Lyapunov exponents for $\tilde{\mathrm{L}}\left(t-t_{0}\right)$ and $\tilde{\mathrm{L}}_{0}\left(t-t_{0}\right)$ is related to $\mathrm{A}-\mathrm{A}_{0}$. Since the difference between $\tilde{\mathrm{L}}\left(t-t_{0}\right)$ and $\tilde{\mathrm{L}}_{0}\left(t-t_{0}\right)$ has an explicit prefactor of $\tilde{\gamma}^{3}$, so will $\mathrm{A}-\mathrm{A}_{0}$. Using the symmetry argument that the Lyapunov exponents have to be even functions of $\gamma$, we obtain

$$
\lambda_{i}+\lambda_{6 N-i+1}=-\alpha_{0}+O\left(\gamma \tilde{\gamma}^{3}\right) . \quad i=1, \ldots 6 N
$$

To explicitly extend the formalism developed in Eqs. (4.19 4.32) to large $t-t_{0}$ and thereby obtain a relation between $\lambda_{i}$ s and $\lambda_{i}^{(0)} \mathrm{s}$, we need to concatenate a lot of $\mathrm{L}(\tau) \mathrm{s}$. These do not commute with each other, nor do they commute with Bs in general. This prevents an explicit demonstration of how the deviation is built up. For the largest and the most negative Lyapunov exponent, it has been possible to show that they pair to $-\alpha_{0}$ plus corrections of $O\left(\gamma \tilde{\gamma}^{3}\right)$ by means of a kinetic theory approach 222,23], based on the independence of subsequent collisions of a sphere. One expects that in subsequent time-intervals of $O\left(\tau_{0}\right)$, the $\mathrm{L}(\tau)$ matrices are not qualitatively much different from each other. Therefore, we expect that the coefficient of the $O\left(\gamma \tilde{\gamma}^{3}\right)$ term in Eq. (4.34), to be of the same order as that for a flight time $\tau=O\left(\tau_{0}\right)$, i.e. of the order of $\mathrm{B}$, which is $O(1)$.

We now return to the discussion of an approximate CPR for the SLLOD equations of motion with an isokinetic Gaussian thermostat in between collisions. As discussed in Sec. III, in the non-equilibrium steady state and in the thermodynamic limit, the coefficient of dynamical friction representing the iso-kinetic Gaussian thermostat for the SLLOD dynamics fluctuates with $1 / \sqrt{N}$ fluctuations around the fixed value $\alpha_{0}$. We would therefore expect that the Lyapunov spectrum for the SLLOD dynamics with an iso-kinetic Gaussian thermostat are given by that of $\mathrm{L}\left(t-t_{0}\right)$ plus terms of $O(1 / \sqrt{N})$. Consequently, the approximate conjugate pairing of Eq. (4.34) can be extended to this thermostat once we neglect the $O(1 / \sqrt{N})$ terms in the sum of the corresponding $\lambda_{i}$ and $\lambda_{6 N-i+1}$.

\section{DISCUSSION}

In this paper, we started with a collection of $N$ hard spheres, each with unit mass and arbitrary radius. Next, we argued how the coefficient of friction representing 
the iso-kinetic Gaussian thermostat in between collisions, fluctuates around a fixed value $\alpha_{0}$ with $1 / \sqrt{N}$ fluctuations in the NESS. Using the properties of the transformation matrices for the infinitesimal phase space element $\delta \boldsymbol{\Gamma}$, we then showed that the CPR is expected to be violated, at the most, at $O\left(\gamma^{4}\right)$ for constant coefficient of friction $\alpha_{0}$. The source of the CPR violation is basically the $\alpha_{0} \gamma \mathbf{C R}$ term in Eq. (2.7), as it is clearly seen that CPR would have been exact in the absence of this term. Finally, we extended that result to the case when the coefficient of friction represents an iso-kinetic Gaussian thermostat. Note that to obtain the deviations from $C P R$ for the constant $\alpha_{0}$ thermostat, the number of particles did not matter, so for that case the result holds for any number of particles, but with an iso-kinetic Gaussian thermostat between collisions, we need to take $N$ to infinity to make the connection to the constant $\alpha_{0}$ thermostat.

In addition, as mentioned in the introduction of this paper, the condition that the mass of each sphere be unity, is not necessary. If we assume that the mass of the $i$-th sphere is $m_{i}$, then one can obtain the same equations of motion, Eq. (2.1 2.2) and (2.7 2.9) in terms of the primed quantities defined by $\mathbf{r}_{i}^{\prime}=\sqrt{m_{i}} \mathbf{r}, \mathbf{v}_{i}^{\prime}=\sqrt{m_{i}} \mathbf{v}$, $\mathbf{p}_{i}^{\prime}=\mathbf{p}_{i} / \sqrt{m_{i}}, \mathbf{R}^{\prime}=\left(\mathbf{r}_{1}^{\prime}, \mathbf{r}_{2}^{\prime}, \ldots, \mathbf{r}_{N}^{\prime}\right), \mathbf{V}^{\prime}=\left(\mathbf{v}_{1}^{\prime}, \mathbf{v}_{2}^{\prime}, \ldots, \mathbf{v}_{N}^{\prime}\right)$ and $\hat{\mathbf{N}}_{i j}^{\prime}$, defined as

$$
\hat{\mathbf{N}}_{i j}^{\prime}{ }^{l}=\left(\delta_{l, i} \sqrt{\frac{m_{j}}{m_{i}+m_{j}}}-\delta_{l, j} \sqrt{\frac{m_{i}}{m_{i}+m_{j}}}\right) \hat{\mathbf{n}}_{i j}
$$

$(l=1,2, . ., N)$. It is then straightforward, albeit lengthy and laborious, to see that our entire analysis goes through in terms of these primed variables, once we use $\mathrm{U}^{\prime}$ in Eq. (A14) with

$$
\begin{aligned}
& \mathrm{U}_{i j}^{\prime}=\mathrm{U}_{j i}^{\prime}=\left[m_{i}+m_{j}\right]^{-1 / 2} \mathrm{I}, \\
& \mathrm{U}_{i i}^{\prime}=\frac{m_{j}}{m_{i}} \mathrm{U}_{j j}^{\prime}=-\sqrt{\frac{m_{j}}{m_{i}}} \mathrm{U}_{i j}^{\prime} .
\end{aligned}
$$

However, we must note that even though the analysis presented in Secs. IV is not affected when the masses and the radii of the spheres are not necessarily the same, one should not allow extreme variations in masses and the radii of the spheres. For large variation of the masses, the system may phase separate into phases in which the mean flight times are different, which will invalidate the use of the Boltzmann equation in Ref. [16] (the Boltzmann equation has been used to show the approach of $\alpha$ towards the constant value $\alpha_{0}$ in Ref. [16]). Furthermore, the use of a typical mean free time in Sec. IV.B will not be possible for large variation in the radii of the spheres. Our analysis in this paper therefore, holds for somewhat limited variation of the masses and the radii of the spheres.

Another possible generalization of our analysis can be carried out for the case when the gas particles interact with each other by means of a short-ranged, repulsive, inter-particle potential (an attractive potential may cause bound states) for a constant multiplier thermostat. The dynamics of the particles can be again decomposed into flights and "collisions" at low density. While the transformation of the infinitesimal phase space volume element over flights will again be determined by the $\mathrm{H}$ matrices as in Eqs. 4.2 4.3) (and thereby have the same properties as above), the corresponding $\mathrm{M}_{i j}$ matrices will not necessarily have a similar form and properties as presented in Eqs. 4.124 .13 . For a given average value of the peculiar kinetic energy, the constant multiplier thermostat has to be chosen carefully (see the last paragraph of Sec. III). Having denoted the constant coefficient of friction again by $\alpha_{0}$, where $\alpha_{0} \propto \gamma^{2}$, is easy to see that the resulting matrix $\mathrm{M}_{i j}$ can be decomposed into a sum of two matrices, $\mathrm{M}_{i j}^{(0)}$ and $\Delta \mathrm{M}_{i j}$, where $\mathrm{M}_{i j}^{(0)}$ is exactly $\mu$-symplectic but $\Delta \mathrm{M}_{i j}$ is not. If the term $\alpha_{0} \gamma \mathbf{C R}$ in Eq. (2.7) (which is the culprit for the violations of CPR in the first place) had been absent, then $\mathrm{M}_{i j}$ would have been $\mu$-symplectic [24] and $\Delta \mathrm{M}_{i j}$ would have been zero, but with Eq. (2.7) in its present form, it is easily seen that $\Delta \mathrm{M}_{i j}$ is $\propto \alpha \gamma \sim \tilde{\gamma}^{3}$ and the proportionality constant depends on the ratio of the time the two colliding particles spend in contact with each other to the time of a flight. This ratio is very small at low density of the gas and therefore the elements of $\Delta \mathrm{M}_{i j}$ are small in comparison with $\mathrm{M}_{i j}^{(0)}$. Since the $\mathrm{H}$ matrices have a similar property, one can repeat the analysis of this paper for such inter-particle short-range repulsive potentials using $\mathrm{H}_{0}$ and $\mathrm{M}_{i j}^{(0)}$ as the reference matrices and arrive at the same conclusion as Eq. 4.34).

Moreover, on the basis of the fact that at low densities and short-ranged repulsive interparticle potentials (that do not give rise to bound states), the constant multiplier thermostat and an iso-kinetic Gaussian thermostat are equivalent in the thermodynamic limit [16], one would expect that under such conditions, the Lyapunov exponent spectrum for the Gaussian thermostat would be practically the same as that of the corresponding constant multiplier thermostat, where the constant multiplier is once again proportional to $\gamma^{2}$. Therefore, in view of the discussion in the previous paragraph, we can also expect $O\left(\tilde{\gamma}^{4}\right)$ deviations from the CPR for a Gaussian thermostatted SLLOD equations of motion in the thermodynamic limit, where the particles interact with each other by means of a short-ranged repulsive potential.

Finally, we note here that verifications of our theory by means of computer simulation remains a challenge. The work for simulations is in progress at present, although our preliminary experience suggests that to retrieve the $\gamma \tilde{\gamma}^{3}$ scaling of the deviation of the sum of the pairs of Lyapunov exponents from $-\alpha_{0}$ is not an easy task.

\section{ACKNOWLEDGMENT}

The authors wish to thank Prof. J. R. Dorfman and Prof. H. van Beijeren for many useful and motivating discussions regarding this subject. D. P. was supported 
by the research grants from "Fundamenteel Onderzoek der Materie (FOM)" and that of Prof. J. R. Dorfman, NSF-PHY-9600428, and wishes to acknowledge the kind hospitality of Prof. H. van Beijeren at University of Utrecht during this and related work. R. v. Z. was supported by the research grant of Prof. H. van Beijeren, by FOM, SMC and NWO Priority Program Non-Linear Systems, which are financially supported by the "Nederlandse Organisatie voor Wetenschappelijk Onderzoek (NWO)", and by a grant from the Natural Sciences and Engineering Research Council of Canada.

\section{APPENDIX:}

In this appendix, we will derive the transformation $\mathrm{M}_{i j}$ of tangent vectors at a collision. The essential element is that the collision does not happen at the same time on reference and on the adjacent trajectory. To understand the origin of this time lag between the binary collisions between the $i$-th and the $j$-th sphere on the reference and the adjacent trajectories, in Fig. 1, we have depicted an exaggerated schematic picture of the collisions taking place in the $6 N$-dimensional phase space on the reference and the adjacent trajectories at points $\mathrm{A}$ and $\mathrm{C}$ respectively. The points $\mathrm{B}$ and $\mathrm{D}$ show the corresponding positions of the adjacent and the reference points respectively when the binary collisions at $\mathrm{A}$ and $\mathrm{C}$ are taking place. Thus, the pre-collisional separation between the reference and the adjacent points is $\overrightarrow{\mathrm{AB}}=\delta \boldsymbol{\Gamma}_{-}$, while the post-collisional separation is $\overrightarrow{\mathrm{DC}}=\delta \boldsymbol{\Gamma}_{+}$. Using that $\left|\mathbf{r}_{j}-\mathbf{r}_{i}\right|$ and $\left|\mathbf{r}_{j}+\delta \mathbf{r}_{j}+\mathbf{v}_{j} \delta \tau-\mathbf{r}_{i}-\delta \mathbf{r}_{i}-\mathbf{v}_{i} \delta \tau\right|$ both have to equal $a_{i}+a_{j}$, the time lag $\delta \tau$ between the two collisions at $\mathrm{A}$ and $\mathrm{C}$, therefore, can be easily expressed as

$$
\delta \tau=-\frac{\left(\delta \mathbf{r}_{j-}-\delta \mathbf{r}_{i-}\right) \cdot \hat{\mathbf{n}}_{i j}}{\left(\mathbf{v}_{j-}-\mathbf{v}_{i-}\right) \cdot \hat{\mathbf{n}}_{i j}}=-\frac{\delta \mathbf{R}_{-} \cdot \hat{\mathbf{N}}_{i j}}{\mathbf{V}_{-} \cdot \hat{\mathbf{N}}_{i j}} .
$$

To obtain the expression of $\delta \boldsymbol{\Gamma}_{+}$, we first express the transformation of $(\mathbf{R}, \mathbf{V})$ in Eq. 2.9) over a binary collision between the $i$-th and the $j$-th sphere in a matrix form

$$
\boldsymbol{\Gamma}_{+}=\mathrm{Q}\left(\boldsymbol{\Gamma}_{-}\right)=\left[\begin{array}{c}
\mathbf{R}_{-} \\
\mathbf{V}_{-}-2\left(\mathbf{V}_{-} \cdot \hat{\mathbf{N}}_{i j}\right) \hat{\mathbf{N}}_{i j}
\end{array}\right]
$$

Next we notice that

$$
\delta \boldsymbol{\Gamma}_{+}=\frac{\partial Q}{\partial \boldsymbol{\Gamma}_{-}} \cdot \delta \boldsymbol{\Gamma}_{-}^{*}-\dot{\boldsymbol{\Gamma}}_{+} \delta \tau
$$

where, $\delta \boldsymbol{\Gamma}_{-}^{*}$ is the infinitesimal phase space separation between the two trajectories at $\mathrm{A}$ and $\mathrm{C}$ is

$$
\delta \boldsymbol{\Gamma}_{-}^{*}=\delta \boldsymbol{\Gamma}_{-}+\dot{\boldsymbol{\Gamma}}_{-} \delta \tau
$$

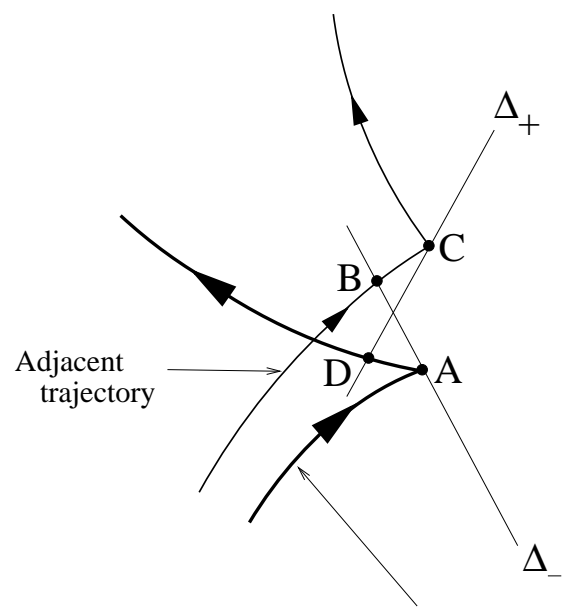

Reference trajectory

FIG. 1. A schematic diagram of the collision dynamics on the reference and the adjacent trajectories.

and $\dot{\boldsymbol{\Gamma}}_{ \pm}$describes the equations of motion, Eq. (2.7), right after(before) the collision at A, i.e.,

$$
\dot{\boldsymbol{\Gamma}}_{ \pm}=\left[\begin{array}{c}
\dot{\mathbf{R}}_{ \pm} \\
\dot{\mathbf{V}}_{ \pm}
\end{array}\right]=\left[\begin{array}{c}
\mathbf{V}_{ \pm} \\
\alpha_{0} \gamma \mathbf{C R}_{ \pm}-\alpha_{0} \mathbf{V}_{ \pm}
\end{array}\right]
$$

Having calculated the quantity $\frac{\partial \mathrm{Q}}{\partial \boldsymbol{\Gamma}_{-}}$from Eq. (A2),

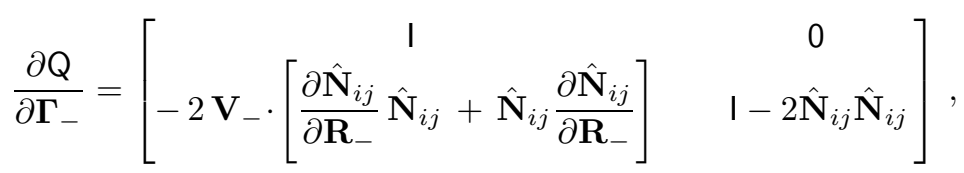

where each entry of the matrix on the r.h.s. of Eq. (A6) is a $3 N \times 3 N$ matrix, the expressions for $\delta \mathbf{R}_{+}$and $\delta \mathbf{V}+$ can be obtained from Eq. A3 as

$$
\delta \mathbf{R}_{+}=\delta \mathbf{R}_{-}-2\left(\delta \mathbf{R}_{-} \cdot \hat{\mathbf{N}}_{i j}\right) \hat{\mathbf{N}}_{i j}
$$

and

$$
\delta \mathbf{V}_{+}=\mathbf{A} \cdot \delta \mathbf{R}_{-}^{*}+\left(\mathbf{I}-2 \hat{\mathbf{N}}_{i j} \hat{\mathbf{N}}_{i j}\right) \delta \mathbf{V}_{-}+\left(\mathbf{I}-2 \hat{\mathbf{N}}_{i j} \hat{\mathbf{N}}_{i j}\right)\left[\alpha_{0} \gamma \mathbf{C} \mathbf{R}_{-}-\alpha_{0} \mathbf{V}_{-}\right] \delta \tau-\left[\alpha_{0} \gamma \mathbf{C} \mathbf{R}_{+}-\alpha_{0} \mathbf{V}_{+}\right] \delta \tau
$$

where $\mathbf{A}=-2 \mathbf{V}_{-} \cdot\left[\frac{\partial \hat{\mathbf{N}}_{i j}}{\partial \mathbf{R}_{-}} \hat{\mathbf{N}}_{i j}+\hat{\mathbf{N}}_{i j} \frac{\partial \hat{\mathbf{N}}_{i j}}{\partial \mathbf{R}_{-}}\right]$and $\delta \mathbf{R}_{-}^{*}=\left(\delta \mathbf{R}_{-}+\mathbf{V}_{-} \delta \tau\right)$. At this point, we use Eq. 2.9$)$ and obtain 


$$
\left(\mathbf{I}-2 \hat{\mathbf{N}}_{i j} \hat{\mathbf{N}}_{i j}\right)\left[\alpha_{0} \gamma \mathbf{C} \mathbf{R}_{-}-\alpha_{0} \mathbf{V}_{-}\right] \delta \tau-\left[\alpha_{0} \gamma \mathbf{C R}_{+}-\alpha_{0} \mathbf{V}_{+}\right] \delta \tau=-2 \alpha_{0} \gamma\left(\hat{\mathbf{N}}_{i j} \cdot \mathbf{C} \mathbf{R}_{-}\right) \hat{\mathbf{N}}_{i j} \delta \tau .
$$

Following Appendix B of [1], the term A $\delta \mathbf{R}_{-}^{*}$ can be expressed as

$$
\mathrm{A} \cdot \delta \mathbf{R}_{-}^{*}=-2\left[\left(\mathbf{V}_{-} \cdot \delta \mathbf{N}_{i j}\right) \hat{\mathbf{N}}_{i j}+\left(\mathbf{V}_{-} \cdot \hat{\mathbf{N}}_{i j}\right) \delta \mathbf{N}_{i j}\right]
$$

where

$$
\delta \mathbf{N}_{i j}=\frac{1}{\sqrt{2}}\left(\mathbf{0}, \mathbf{0}, \ldots, \delta \mathbf{n}_{i j}, \ldots-\delta \mathbf{n}_{i j}, \ldots \mathbf{0}\right),
$$

satisfying $\hat{\mathbf{n}}_{i j} \cdot \delta \mathbf{n}_{i j}=0$. This orthogonality condition between $\hat{\mathbf{n}}_{i j}$ and $\delta \mathbf{n}_{i j}$ also implies that $\hat{\mathbf{N}}_{i j} \cdot \delta \mathbf{N}_{i j}=0$. To obtain an expression for $\delta \mathbf{n}_{i j}$, we need to take a look at Figs. 2 and 3 . Figure 2 describes, in the laboratory frame, the binary collision process between the $i$-th and the $j$-th sphere on the reference and adjacent trajectories; the thick-lined spheres are on the reference trajectory whereas the thin-lined spheres are on the adjacent trajectory. Figure 3 describes the same binary collision process in the reference frame of the $i$-th sphere (with center C). In Fig. 2, the thick-lined $j$-th sphere (with center D) on the left depicts the collision situation on the reference trajectory and the thin-lined $j$-th sphere (with center E) on the left depicts the collision situation on the adjacent trajectory.

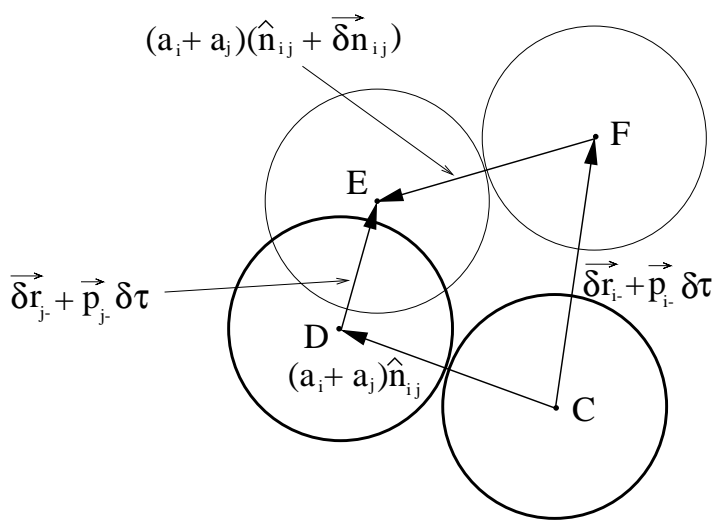

FIG. 2. Collision between the $i$-th and the $j$-th sphere. Thick-lined spheres are on the reference trajectory whereas the thin-lined spheres are on the adjacent trajectory.
Clearly, in Fig. 3, the infinitesimal vector $\overrightarrow{\mathrm{DE}}$ is given by

$$
\delta \mathbf{r}_{i j}^{*}=\delta \mathbf{r}_{j-}-\delta \mathbf{r}_{i-}+\left(\mathbf{v}_{j-}-\mathbf{v}_{i-}\right) \delta \tau
$$

and since the lengths of both the lines $\mathrm{CD}$ and $\mathrm{CE}$ are $a_{i}+a_{j}\left(a_{i}\right.$ and $a_{j}$ are the radii of the $i$-th and the $j$-th sphere respectively), we have

$$
\delta \mathbf{n}_{i j}=\frac{1}{a_{i}+a_{j}} \delta \mathbf{r}_{i j}^{*}
$$

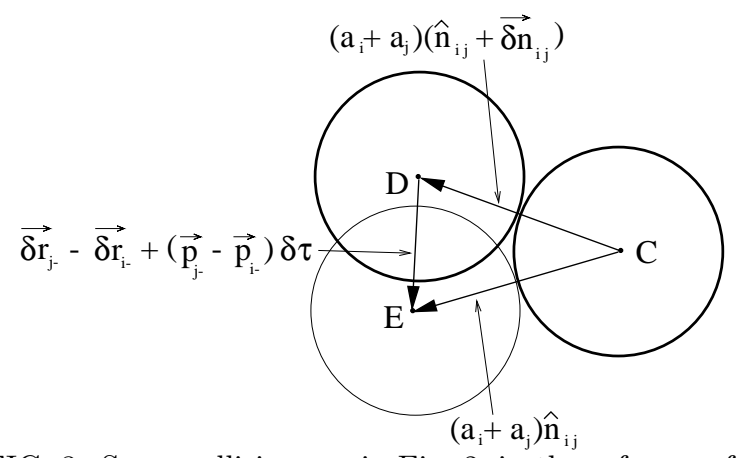

FIG. 3. Same collisions as in Fig. 2, in the reference frame of the $i$-th sphere.

Let us define a $3 N \times 3 N$ matrix $\mathrm{U}$ composed of $N \times N$ blocks of $3 \times 3$ matrices, such that, in terms of the block indices the only non-zero entries of $U$ are

$$
\mathrm{U}_{i i}=-\mathrm{U}_{i j}=-\mathrm{U}_{j i}=\mathrm{U}_{j j}=-\mathrm{I},
$$

where I is the $3 \times 3$ unit matrix. One can now write, using Eqs. (A11-A14) and Eq. (A1) that

$$
\delta \mathbf{N}_{i j}=\frac{1}{\sqrt{2}\left(a_{i}+a_{j}\right)}\left[\mathbf{U} \cdot \delta \mathbf{R}_{-}+\mathbf{U} \cdot \dot{\boldsymbol{\Gamma}}_{-} \delta \tau\right]=\frac{1}{\sqrt{2}\left(a_{i}+a_{j}\right)}\left[\mathbf{U}-\frac{\left(\mathrm{U} \cdot \mathbf{V}_{-}\right) \hat{\mathbf{N}}_{i j}}{\mathbf{V}_{-} \cdot \hat{\mathbf{N}}_{i j}}\right] \cdot \delta \mathbf{R}_{-} .
$$

From Eqs. A10 and Eq. A15, we finally have

$$
\begin{aligned}
\mathbf{A} \cdot \delta \mathbf{R}_{-}^{*} & =\frac{\sqrt{2}}{a_{i}+a_{j}}\left[\left(\mathbf{U} \cdot \mathbf{V}_{-}\right) \hat{\mathbf{N}}_{i j}+\hat{\mathbf{N}}_{i j}\left(\mathbf{U} \cdot \mathbf{V}_{-}\right)-\left(\hat{\mathbf{N}}_{i j} \cdot \mathbf{V}_{-}\right) \mathrm{U}+\frac{\mathbf{V}_{-} \cdot \mathbf{U} \cdot \mathbf{V}_{-}}{\mathbf{V}_{-} \cdot \hat{\mathbf{N}}_{i j}} \hat{\mathbf{N}}_{i j} \hat{\mathbf{N}}_{i j}\right] \cdot \delta \mathbf{R}_{-} \\
& =\left(\mathrm{I}-2 \hat{\mathbf{N}}_{i j} \hat{\mathbf{N}}_{i j}\right) \mathrm{W} \cdot \delta \mathbf{R}_{-} .
\end{aligned}
$$


where $\mathrm{W}$ is a $3 N \times 3 N$ symmetric matrix. Finally, using Eqs. A1, (A7 A9) and (A16), the expression for $\mathrm{M}_{i j}$ can be obtained as

$$
\mathrm{M}_{i j}=\left(\mathrm{I}-2 \hat{\mathbf{N}}_{i j} \hat{\mathbf{N}}_{i j}\right)\left[\begin{array}{ll}
\mathrm{I} & 0 \\
\mathrm{R} & \mathrm{I}
\end{array}\right],
$$

where

$$
\mathrm{R}=\mathrm{W}-2 \alpha_{0} \gamma \frac{\hat{\mathbf{N}}_{i j} \cdot \mathbf{C} \mathbf{R}_{-}}{\mathbf{V}_{-} \cdot \hat{\mathbf{N}}_{i j}} \hat{\mathbf{N}}_{i j} \hat{\mathbf{N}}_{i j}
$$

[1] A. W. Lees and S. F. Edwards, J. Phys. C 5, 1921 (1972).

[2] W. G. Hoover, D. J. Evans, R. B. Hickman, A. J. Ladd, W. T. Ashurst, and B. Moran, Phys. Rev. A 22, 1690 (1980).

[3] D. J. Evans and G. P. Morriss, Comp. Phys. Rep. 1, 297 (1983); Ibid., Phys. Rev. A 30, 1528 (1984).

[4] D. J. Evans and G. P. Morriss, Statistical Mechanics of Non-equilibrium Liquids, Academic Press, London, 1990.

[5] G. P. Morriss, Phys. Lett. 134A, 307 (1989).

[6] D. J. Evans, E. G. D. Cohen and G. P. Morriss, Phys. Rev. A, 42, 5990 (1990).

[7] S. Sarman, D. J. Evans and G. P. Morriss, Phys. Rev. A 45, 2233 (1992).

[8] C. P. Dettmann and G. P. Morriss, Phys. Rev. E 53, R5545 (1996).

[9] M. P. Wojtkowski and C. Liverani, Comm. Math. Phys. 194, 47 (1998).

[10] D. Ruelle, J. Stat. Phys. 95, 393 (1999).
[11] D. Panja, An Elementary Proof of Lyapunov Exponent Pairing for Hard-Sphere Systems at Constant Kinetic Energy preprint hlin.CD/0107048.

[12] D. J. Isbister, D. J. Searles and D. J. Evans, Physica A 240, 105 (1997).

[13] D. J. Searles, and D. J. Evans and D. J. Isbister, Chaos 8, 337 (1998).

[14] G. P. Morriss, Phys. Rev. E 65, 17201 (2002).

[15] In reality, turbulence develops if the system's dimension in the direction on the gradient of velocity becomes too large, but the iso-kinetic and the constant $\alpha$ thermostats stabilize the linear flow field, even for $N \rightarrow \infty$.

[16] R. van Zon, Phys. Rev. E 60, 4158 (1999).

[17] J. Petravic, D. J. Isbister, and G. P. Morriss, J. Stat. Phys. 76, 1045 (1994).

[18] P. Gaspard and J.R. Dorfman, Phys. Rev. E 52, 3525 (1995).

[19] This condition has been quoted as a necessary condition for an exact CPR in Ref. [13], but it is actually a sufficient condition.

[20] Ch. Dellago, H. A. Posch and W. G. Hoover, Phys. Rev. E 53, 1485 (1996).

[21] Here we assume that such a power series expansion can be carried out for individual Lyapunov exponents. This is not unreasonable, but it might be applicable only to low densities. In any case, it is clear from the previous paragraph that the presence of such non-analytic terms can only give rise to differences between the finite-time Lyapunov exponents $\lambda_{i}(\tau)$ and $\lambda_{i}^{(0)}(\tau)$ (or the actual Lyapunov exponents $\lambda_{i}$ and $\left.\lambda_{i}^{(0)}\right)$ that are weaker than $\gamma \tilde{\gamma}^{2}$.

[22] R. van Zon, Ph.D. thesis, University of Utrecht, 2000.

[23] R. van Zon and H. van Beijeren (unpublished).

[24] U. Dressler, Phys. Rev. A 38, 2103 (1988). 